\section{Neues aus den Hochschulen}

\section{Fortbildungsseminar GIS in München}

Der Runde Tisch GIS e.V. und die Technische Universität München veranstalten gemeinsam vom 12.-14. März 2003 das 8. Münchner Fortbildungsseminar Geoinformationssysteme an der TU München.

Die Themen des dreitägigen Seminars sind:

- Geoinformationssysteme und Location Based Services (Tutorial)

- Best-Practice Projekte: Ausgewählte innovative Projekte

- OpenGIS im Praxistest - Interoperable Datennutzung im Internet

- Spezialthema: Datensicherheit im Internet.

Anlässlich des Fortbildungsseminars wird der Förderpreis Geoinformatik 2003 des Runden Tisches GIS e.V. verliehen.

Der aktuelle Stand des Programms kann auf den Seiten des Runden Tisches GIS e.V. nachgelesen werden:

http://www.rundertischgis.de

Weitere Informationen: Technische Universität München, Fachgebiet Geoinformationssysteme, Frau Horvath, Tel. 089 2892-2578,eva.horvath@bv.tum.de

\section{Karten grenzenlos skalier- bar - Workshop Internet- Kartographie mit SVG}

Mit dem Vektorformat SVG können anspruchsvolle interaktive Kartenprojekte im Internet realisiert werden. Das Ziel des Workshops ist es, diese Möglichkeiten kennenzulernen und einzuüben, um sie anschließend selbstständig in eigenen Projekten zu nutzen. Die Veranstaltung richtet sich dabei an alle, die bisher keine oder wenig Erfahrung haben, vektororientierte Karten im Internet zu prösentieren. Die grundlegenden Arbeitsweisen an einem PC sollten jedem vertraut sein. Vorkenntnisse in Bezug auf das Internet und den damit verbundenen Techniken sind nicht notwendig.

Folgende Inhalte werden behandelt:

- Einführung in das Webmapping

- Vektorgrafik: Umfeld und Entwicklungen

- Erstellen von Internet-fähigen Karten in SVG

- Interaktivität und Animation mit SVG (und JavaScript)

- Integrieren von Geometrien aus GIS und Graphik-Programmen

- Datenbanken und SVG.

Der Workshop findet vom 10. bis 14. Februar 2003 an der Technische Fachhochschule Berlin (Studiengang Kartographie) statt. Die Teilnahmegebühr beträgt 490,- $€$, bei einer Anmeldung bis 20.12.2002 ermäßigt sich die Gebühr auf 440,- $€$. Anmeldeschluss ist der 24. Januar 2003.

Leitung:

Prof. Dr. Jürgen Schweikart (TFH Berlin)

Referenten:

Dipl.-Ing. Nicole Ueberschär (TFH Berlin) Mag. André M. Winter (Universität Innsbruck)

Anmeldung und Information:

TFH Berlin, Luxemburger Straße 10, D-13353 Berlin, Tel.: (030) 4504-2607, -2613,-2038, Email: veberschuer@ thh-berlin.de, schweikart@tfh-berlin.de Website zum Workshop: http://svg-kurs. carto.net

\title{
Neues aus der DGfK
}

\section{DGfK-Kommission "Karto- graphische Terminologie"}

Bericht über die Arbeiten der Kommission seit dem Deutschen Kartographentag im Oktober 2001

\section{Kommissionsmitglieder}

Gegenüber dem letzten Jahr haben sich hier keine Veränderungen ergeben (vgl. KT 2001, S. 97/98). An der Arbeit waren jedoch auch weitere Fachkollegen, insbesondere der Universitüten Dresden und Trier, beteiligt, bedingt durch die Bearbeitung des umfangreichen neven Kartographielexikons. In die Endarbeiten zum F.I.G.Wörterbuch, Band „Kartographie”, wurde die Dresdner Studentin R. Riedel, die gleichfalls nicht zur Kommission gehört, einbezogen.

Kommissionssitzungen, Workshops Vollsitzungen der Kommission haben am 4.10.2001 in Berchtesgaden im Rahmen des 50. Deutschen Kartographentages und am 28.6.2002 in Leipzig stattgefunden. Ein Workshop wurde nach Abschluss der Arbeiten am "Lexikon der Kartographie und Geomatik" am 28.6.2002 gemeinsam mit dem Institut für Länderkunde Leipzig und den Universitäten Dresden und Trier in Leipzig durchgeführt.
Ergebnisse der Arbeit 2001/2002 Auf dem 50. Deutschen Kartographentag in Berchtesgaden hat der Leiter der Kommission die bis dahin erzielten Arbeitsergebnisse vorgestellt. Dabei wurde insbesondere die oft recht mühselige, aber immer wieder von der Ausdauer und vom Optimismus der Kommissionsmitglieder getragene Arbeit am Hauptprojekt, dem "Lexikon der Kartographie und Geomatik", seit der Auftragserteilung durch den Präsidenten der DGfK, Prof. Dr. U. Freitag, geschildert. Der erste Band der Lexikons (A bis Karti, 450 Seiten) konnte von einer Vertreterin des Spektrum Akademischer Verlag in Berchtesgaden an den Kommissionsleiter übergeben werden.

Bis in den Januar 2002 hinein wurde intensiv weiter am zweiten Band gearbeitet, wobei von den Kommissionsmitgliedern primär W. G. Koch (Herausgeber) und W. Stams, von den weiteren Lexikonmitarbeitern vorrangig J. Bollmann (Herausgeber), A.-D. Uthe und die Kollegen der Abt. Kartographie der Universität Trier beteiligt waren. Zahlreiche wichtige Stichworttexte waren noch zu schreiben, auch Kürzungen vorzunehmen, Bilder, insbesondere Farbbilder, zu beschaffen und zu bearbeiten sowie redaktionelle Endarbeiten auszuführen. An den letzteren war A. Lipinski

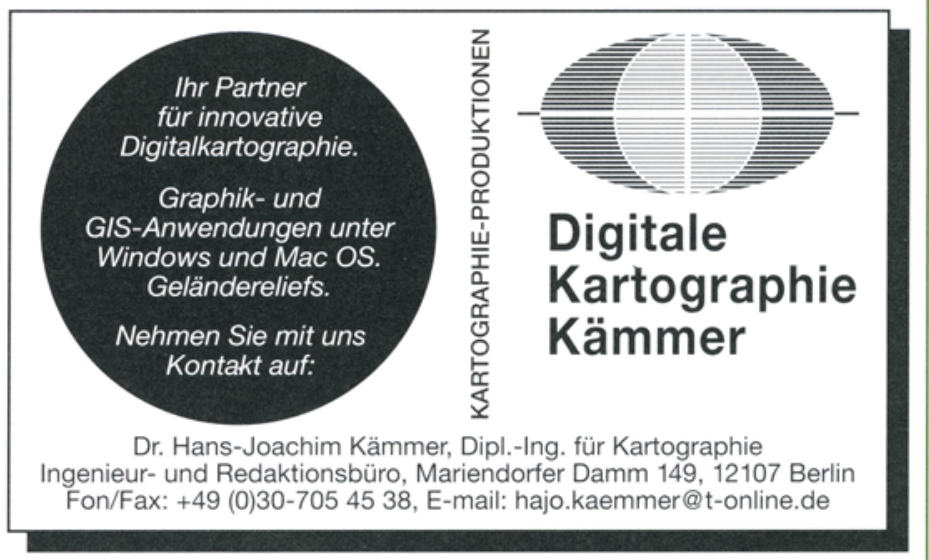

